data warehouses to deliver EHR data provides a valuable tool for completing retrospective precision medicine projects. The validation of definitions for clinical outcomes identifiable retrospectively are necessary and provide novel guidance for future studies.

4192

\section{Decreasing Inappropriate STAT Image Ordering}

Michael Cui ${ }^{1}$, Jonathan Chung ${ }^{1}$, Pritesh Patel ${ }^{1}$, and Ingrid Reiser ${ }^{1}$ ${ }^{1}$ University of Chicago

OBJECTIVES/GOALS: Currently physicians are able to order CT Chest/Abd/Pelvis images as STAT or Routine. STAT images denote an emergency and are done immediately. We aim to determine the percentage of CT images that are inappropriately ordered as STAT, determine physician image ordering habits, and develop targeted interventions to encourage appropriate STAT image ordering. METHODS/STUDY POPULATION: A fishbone diagram helped reveal possible causes of inappropriate STAT image ordering. Based on the fishbone diagram, a survey was created to assess CT image ordering habits amongst radiology and internal medicine residents and attending physicians. All CT Chest/Abd/Pelvis images ordered over a 3 month period of time (July-Oct 2017) was obtained. The dataset included whether the image was ordered Stat vs Routine, time of image order, physician name and location, and reason for the imaging study.The STAT images were evaluated based on the explanation provided in the CT image order. Currently 2 radiology residents, 2 internal medicine residents, and 2 internal medicine hospitalists are evaluating all STAT CT images to determine appropriateness and how long they are willing to wait for the image to result in a read. RESULTS/ ANTICIPATED RESULTS: Analysis of all CT Chest/Abd/Pelvis imaging orders revealed that $51 \%(1710 / 3345)$ of them were ordered as STAT. The preliminary analysis of 227 STAT images showed that $6 \%$ were inappropriate. We anticipate results of our survey to show differences in how long a STAT vs Routine image orders should take amongst Radiology and Internal Medicine clinicians. We also anticipate our survey to show differences in factors that warrant STAT imaging amongst the different medical fields. We anticipate that the clinician manual evaluation of all STAT CT image will reveal a large percentage of imaging orders to be inappropriate. All STAT imaging that were flagged as inappropriate will be characterized by the department who ordered the image and the reason provided for the imaging to assess for common themes. DISCUSSION/SIGNIFICANCE OF IMPACT: STAT images are the new routine with more images ordered STAT than Routine. Inappropriate STAT imaging results in truly urgent patients not getting the medical care they need. Many images ordered stat could potentially be switched to routine. By evaluating why clinicians are ordering STAT CT image inappropriately, we will be able to develop targeted interventions to decrease inappropriate STAT CT imaging.

4557

Defining the relationship between kidney structure and function in patients with and without diabetes and hypertension

Ghazal Zekavat Quinn ${ }^{1}$, Matthew Palmer ${ }^{1}$, Jordana Cohen ${ }^{1}$, Xin Sheng ${ }^{1}$, and Katalin Susztak ${ }^{1}$

${ }^{1}$ University of Pennsylvania School of Medicine

OBJECTIVES/GOALS: Histopathological descriptions of kidney tissue provide more information about kidney disease severity and prognosis than serum measures, yet most patients with chronic kidney disease do not undergo kidney biopsy. We aim to develop a method to determine the degree of renal injury in patients with diabetes and hypertension without the need for biopsy. METHODS/ STUDY POPULATION: Clinical data and renal tissue samples were collected from 864 patients undergoing tumor-associated nephrectomy in seven medical centers in the United States. Exclusion criteria included age $<18$, presence of pyelonephritis or non-diabetic or hypertensive renal disease or incomplete clinical or histopathologic data. 19 histologic parameters were scored in a blinded manner by one renal pathologist. We examined the relationship between and functional variables (such as estimated glomerular filtration rate (eGFR)). Polynomial regression analysis was performed to model histopathologic variables and important clinical parameters such as eGFR RESULTS/ANTICIPATED RESULTS: 607 samples met inclusion criteria and were stratified as: control (no history of diabetes or hypertension, $n=160)$, hypertension alone $(n=224)$ and both diabetes and hypertension $(n=223)$. Interstitial fibrosis (IF) and glomerulosclerosis (GS) had the strongest correlations with eGFR. Regression analysis was used to model histopathologic score for a given eGFR. We found that diabetes and hypertension modified the relationship between tubulointerstitial fibrosis and eGFR. For example, while hypertensive patients without diabetes had $33 \%$ IF at an eGFR of $30 \mathrm{ml} / \mathrm{min} / 1.73 \mathrm{~m}^{2}\left(\mathrm{r}^{2}=0.64, \mathrm{p}<0.01\right)$, hypertensive patients with diabetes had $32 \%$ IF at an eGFR of $30 \mathrm{ml} / \mathrm{min} /$ $1.73 \mathrm{~m}^{2}\left(\mathrm{r}^{2}=.43, \mathrm{p}<0.01\right)$ and control patients had approximately $23 \%$ IF at an eGFR of $30 \mathrm{ml} / \mathrm{min} / 1.73 \mathrm{~m}^{2}\left(\mathrm{r}^{2}=0.22, \mathrm{p}<0.01\right)$. DISCUSSION/SIGNIFICANCE OF IMPACT: Here, we describe the relationship between renal structural changes and renal function and show that hypertension significantly modifies the relationship at a given eGFR. These data can be used to reasonably predict renal structural changes given clinical information without the need for renal biopsy and may provide prognostic value.

4219

Discrepancies in flavor preferences among adult ever users of various tobacco products in the US - Findings from The Population Assessment of Tobacco and Health Study (2015-2016)

Liane M Schneller ${ }^{1}$, Dongmei Li ${ }^{1}$, Zahíra Quiñones-Tavárez ${ }^{1}$, Maciej Goniewicz $^{1}$, Amanda Quisenberry ${ }^{1}$, Zidian Xie ${ }^{1}$, Irfan Rahman $^{1}$, Scott McIntosh ${ }^{1}$, Richard O'Connor ${ }^{1}$, and Deborah J. Ossip, PhD ${ }^{1}$ ${ }^{1}$ University of Rochester Medical Center

OBJECTIVES/GOALS: Flavorings differ between brands and tobacco products, potentially altering the sensory perceptions. This study aimed to examine discrepancies in flavor preference across various noncigarette tobacco products among a national representative sample of US adult regular tobacco users. METHODS/STUDY POPULATION: Data from the Population Assessment of Tobacco and Health (PATH) Study Wave 3 (W3) were used. Weighted prevalence of flavor preference for various tobacco products, including electronic nicotine delivery systems (ENDS), traditional cigars, cigarillos/ filtered cigars, hookah and snus/smokeless, was presented for 9,037 adult current and new former users of multiple flavored tobacco products. Within-subject flavor discrepancies were assessed using generalized estimating equations (GEE) models considering the complex sampling design of the PATH study. RESULTS/ANTICIPATED RESULTS: Most regular users of a flavored tobacco products reported using one flavor category per product. Fruit flavors, followed by tobacco, were the most common flavor categories among ENDS 